\title{
Vibrational Spectroscopies and Chemometry for Nondestructive Identification and Differentiation of Painting Binders
}

\author{
Serena Carlesi, ${ }^{1}$ Maurizio Becucci, ${ }^{1,2}$ and Marilena Ricci ${ }^{1}$ \\ ${ }^{1}$ Department of Chemistry "Ugo Schiff”, University of Florence, Via della Lastruccia 3-13, 50019 Sesto Fiorentino, Italy \\ ${ }^{2}$ The European Laboratory for Non-Linear Spectroscopy (LENS), Via N. Carrara 1, 50019 Sesto Fiorentino (FI), Italy
}

Correspondence should be addressed to Marilena Ricci; marilena.ricci@unifi.it

Received 1 February 2017; Accepted 15 March 2017; Published 25 May 2017

Academic Editor: Ewa Schab-Balcerzak

Copyright (C) 2017 Serena Carlesi et al. This is an open access article distributed under the Creative Commons Attribution License, which permits unrestricted use, distribution, and reproduction in any medium, provided the original work is properly cited.

\begin{abstract}
A comprehensive dataset of vibrational spectra of different natural organic binding media is presented and discussed. The binding media were applied on a glass substrate and analyzed after three months of natural ageing. The combination of Raman and FT-NIR spectroscopies allows for an improved identification of these materials as Raman technique is more informative about the skeletal vibrations, while FT-NIR spectroscopy is more sensitive to the substituents and polar groups. The experimental results are initially discussed in the framework of current spectral assignment. Then, multivariate analysis (PCA) is applied leading to differentiation among the samples. The two major principal components allow for a complete separation of the different classes of organic materials. Further differentiation within the same class is possible thanks to the secondary components. The loadings obtained from PCA are discussed on the basis of the spectral assignment leading to clear understanding of the physical basis of this differentiation process.
\end{abstract}

\section{Introduction}

The chemical nature of the materials used in painting as binding media is usually very diverse and complex, which makes their characterization a challenging task. A greater knowledge of the original binders used by the artist is of great interest to art historians because it allows them to distinguish between different painting techniques, which are often directly associated with the geographical, social, and chronological contexts in which the artist lived and worked. Information about the class of binders is also important for restorers, who often use solvents during the cleaning and restoration of artworks [1].

The identification and chemical characterization of various painting binders (protein-, polysaccharide-, and lipidbased) has been frequently addressed in published research, mainly using techniques such as gas chromatography/mass spectrometry (GC/MS) in combination with various offline or online derivatization procedures $[2,3]$. Recent authors have proposed the use of spectroscopic techniques, as vibrational and electronic spectroscopic methods, coupled with multivariate analysis for the discrimination of different painting binders (animal glue, whole egg, and linseed oil) [2, 4-6] and also of organic compounds of the same class, like lipidic binders [7-10], subjected to natural ageing.
Separation methods (like gas chromatography, capillary electrophoresis, and liquid chromatography) and mass spectrometry present limitations for their application, such as large sample consumption, long experimental times, complex sample preparation, and multiparameter operating conditions. These are obvious problems for the analysis of samples of historical interest $[4,11,12]$. Spectroscopic techniques have the potential to overcome these limitations, as they allow nondestructive and contactless measurements, giving molecular information on complex macromolecules, and could be performed in situ by means of portable devices.

The work presented here explores the application of chemometric tools, such as principal component analysis (PCA) to combined FT-NIR and Raman data in order to enable us to distinguish among organic compounds of the same and different classes. With this aim in mind, we prepared 13 model samples containing traditional organic materials, commonly used by artists throughout history as binding media. These models samples were ageing naturally for three months at room temperature, without controlled environmental conditions, and then were further characterized by Raman and FTNIR spectroscopy. The present model is not a realistic presentation of fully aged artworks, because the ageing processes of 
these materials last a much longer time. Nevertheless these models are useful for assessing the possibility of our data analysis.

Because these two vibrational spectroscopies obey different selection rules and provide often complementary information, combining the two could permit a greater amount of spectroscopic information to be extracted from each sample. Nevertheless, there are several different causes that can lead to random noise in Raman and FT-NIR spectra, thus making the identification of spectral bands more difficult, particularly in computational analyses, as in the present work. To identify small differences in the original spectra, first derivatives of the spectral data were used for PCs extraction $[4,6,8,9$, 13]. As a result, an increase in sensitivity/specificity for differentiation capability and classification can be expected, due to the new dimensions added to the dataset $[8,9]$. Principal Component Analysis (PCA) can be used to explain the variance-covariance structure of a set of data by the original variables' linear combination that form a new set of independent coordinates, called principal components (PC). The PCs are hierarchically ordered to describe the largest variance of the dataset, so that a smaller number of variables can account for most of the information from the original dataset [14]. The purpose of all multivariate data analysis is to decompose the data in order to detect and model the "hidden phenomena" or better to define the set of natural coordinates for these variables that show more clearly the similitudes and differences between the various samples $[14,15]$. Therefore, the application of PCA to large spectral datasets can be exploited to extract meaningful information from spectral differences, allowing us to discriminate among different samples based on their composition $[14,15]$.

The results clearly show the potential of this methodology to extract the information needed to identify the painting materials that is contained in the FT-NIR and Raman spectra.

\section{Materials and Methods}

2.1. Materials. All neat binding media analyzed in this work, along with corresponding symbols, are listed in Table 1.

Each drying oil, as supplied, was applied as film on four microscope glass slides. Proteinaceous and polysaccharide binding media were prepared according to recipes from original treatises and artists' accounts [16-18]. Rabbit skin glue, strong glue, and fish glue were swollen and dissolved in warm demineralized water to give a solution of $5 \%$ (w/w). Egg white was beaten to form stiff peaks and left for $24 \mathrm{~h}$; foam was skimmed and removed and then a solution of $50 \%(\mathrm{w} / \mathrm{w})$ clear egg white in demineralized water was prepared. Egg yolk was extracted from egg by piercing the yolk and allowing the liquid to drip from the encasing film; the yolk was diluted in demineralized water to give a $50 \%(\mathrm{w} / \mathrm{w})$ emulsion. Whole egg was prepared by mixing one part of egg white solution and two parts on egg yolk emulsion along with one part of vinegar. Casein was swollen in water for $24 \mathrm{~h}$ to give a $1.5 \%(\mathrm{w} / \mathrm{w})$ solution; dilute ammonia solution was added until the swollen gel dissolved and excess ammonia was left to evaporate. Arabic gum was dissolved in warm demineralized water with a ratio of 1 to $2(\mathrm{w} / \mathrm{w})$, heating in a double boiler;
TABLE 1: The neat binding media analyzed with reporting the manufacturer and the corresponding identification symbol.

\begin{tabular}{lcc}
\hline Neat binder & Producer/manufacturer & Symbol \\
\hline Proteinaceous binders & & \\
Rabbit skin glue & Zecchi & RG \\
Strong glue & Zecchi & SG \\
Fish glue & Zecchi & FG \\
Egg white & Local market & EW \\
Egg yolk & Local market & EY \\
Whole egg & Local market & WE \\
Casein & Zecchi & C \\
Drying oils & & \\
Linseed oil & Zecchi & LOZ \\
Linseed oil & Maimeri & LOM \\
Linseed oil & Ferrario & LOF \\
Poppy-seed oil & Zecchi & POZ \\
Poppy-seed oil & Talens & POT \\
Walnut oil & Zecchi & WOZ \\
Walnut oil & Ferrario & WOF \\
Stand-oil & Zecchi & SOLZ \\
Polysaccharide media & & \\
Arabic gum & Zecchi & AG \\
Tragacanth gum & Zecchi & TG \\
\hline
\end{tabular}

the mixture was left to soak $48 \mathrm{~h}$ for a full absorption and finally, the obtained solution was strained through two layers of cheesecloth into a clean jar. Tragacanth gum was added to demineralized water in a ratio 1:30 (w/w) and left to swell in a mucilaginous suspension. Then, this suspension was strain in a similar way to that previously described for Arabic gum.

All these organic binders and oils were individually applied as film on four microscope glass slides and left to dry on a shelf ( $T: \sim 20^{\circ} \mathrm{C}$; RH: $\sim 50-55 \%$; light: solar light through a window). Hence, sixty-eight model samples for neat binders were prepared.

These materials were analyzed after an ageing of three months. As regards drying oils, they should be treated as "fresh" binding media because the drying process has not already been completed (according to previous studies, an oil paint can be regarded as aged only one year after its preparation $[17,19])$.

\subsection{Fourier Transform-Near Infrared (FT-NIR) Measure-} ments. FT-NIR reflectance spectra were acquired by using a portable ALPHA FT-IR spectrometer (Bruker Optics) equipped with an external reflection module. Total reflection spectra (including both the specular and diffuse reflection contributions) were collected in situ, in the spectral range $7500-3900 \mathrm{~cm}^{-1}$, with a resolution of $4 \mathrm{~cm}^{-1}$, averaging over 128 scans. The spectral sampling distance used for each scan was $2 \mathrm{~cm}^{-1}$. The background was acquired using a gold mirror as reference sample. In total, 272 spectra were recorded, at four different positions for each sample (16 spectra for each binder). 
2.3. Micro-Raman Spectroscopy. Micro-Raman analysis was performed using a micro-Raman spectrometer (Renishaw 2000) equipped with a $785 \mathrm{~nm}$ diode laser, operating at $1.1 \mathrm{~mW}$ laser power on the sample. The laser was focused onto the sample using a 50x objective lens, so that an area of about $2 \mu \mathrm{m}$ in diameter was analyzed. Spectra were recorded in the $2000-200 \mathrm{~cm}^{-1}$ spectral range, with a spectral resolution of $4 \mathrm{~cm}^{-1}$. The integration time and the spectral sampling distance used for each scan were $25 \mathrm{~s}$ and $1 \mathrm{~cm}^{-1}$, respectively. Each spectrum was the average of three acquisitions. The spectrometer was frequency calibrated at the beginning of each measurement session by using the Raman signal from a silicon wafer at $520 \mathrm{~cm}^{-1}$. No changes related to photochemical damage were observed on the samples after the Raman measurements. In total, 272 spectra were recorded, at four different positions for each sample (16 spectra for each binder).

2.4. Data Analysis. Micro-Raman and FT-NIR spectral datasets were preprocessed using the PCA method by means of The Unscrambler X version 10.3 software (CAMO).

Although micro-Raman spectra were registered between 2000 and $200 \mathrm{~cm}^{-1}$, data analysis was performed in the $1800-750 \mathrm{~cm}^{-1}$ range, since the most important bands of binding media are located in this spectral region. The FTNIR spectra were analyzed in the 6000 and $3900 \mathrm{~cm}^{-1}$ range, where the signal was well defined and not much affected by noise.

The FT-NIR and micro-Raman datasets were preprocessed separately to discern variability in the original spectra. In detail, the FT-NIR spectra were subjected to the SavitzkyGolay derivative algorithm [20] (2nd order polynomial, 15 data points) without any other preprocessing. The microRaman spectra were, instead, subjected to baseline subtraction, average reduction of variables (factor two), and first derivative calculation by applying the Savitzky-Golay algorithm (2nd order polynomial, 15 data points). Then, both the FT-NIR and micro-Raman spectra were vector normalized, as the use of absolute band heights could lead to erroneous sample classifications [7]. The combined spectra were obtained as follows: the first derivative Raman and FT-NIR spectra were joined, generating the new combined Raman-FT-NIR spectrum, 16 for each typology of sample.

Each new combined spectrum includes 1575 data points, with the first 1050 derived from the FT-NIR spectrum abscissa, related to the spectral region $6000-3900 \mathrm{~cm}^{-1}$, and the remaining 525 points taken from the micro-Raman spectrum abscissa in the $1800-750 \mathrm{~cm}^{-1}$ spectral region. Each combined spectrum is characterized by both fundamental and combination-overtone modes, as have been previously described in the FT-NIR and micro-Raman sections.

The Uncertainty Test was always performed to estimate the significance of variables. Then, PCA was performed by applying a mean centering onto data column-wise, and the Nonlinear Iterative Partial Least Squares (NIPALS) algorithm. Each model was always validated by Leave One Out (LOO) cross-validation and by a projection of evaluated sets of spectral data acquired by the same samples (four spectra for each type of sample).

\section{Results and Discussion}

This study involves the application of the Principal Component Analysis (PCA) on FT-NIR and micro-Raman spectroscopic data in order to characterize the spectral differences, in frequency and intensity, among various classes of binding media. The following discussion is divided into two main sections dealing with an initial characterization of FT-NIR and Raman spectra of each binder and a successive evaluation of results obtained from the statistical model.

3.1. FT-NIR and Micro-Raman Spectra of Different Binders. First, we discuss the proteinaceous binders such as casein, egg white, and glues; following, we analyze the egg yolk and whole egg, which differ from the other protein-based binding media for the lipid content $[6,18]$. Finally we discuss the spectra of the other materials.

We report in Figure 1 the FT-NIR spectra (Figure 1(a)) and the Raman spectra (Figure 1(b)) of casein, egg white, and glues. The raw FT-NIR spectra of these proteinaceous binding media do not show significant spectral differences. The combination band of amide III (C-N stretching/ $\mathrm{N}-\mathrm{H}$ in plane bending), the first overtone of carbonyl stretching $\left(\sim 4600 \mathrm{~cm}^{-1}\right)$, and the combination band of the stretching and bending modes of $\mathrm{NH}\left(\sim 4880 \mathrm{~cm}^{-1}\right)$ represent the characteristic signals of proteins. Furthermore, a weak absorption band, located at about $3988 \mathrm{~cm}^{-1}$ and ascribed to the first C$\mathrm{N}-\mathrm{C}$ stretching overtone in amides, can be observed $[6,21]$. For all these binders, the methylenic overtones and combination bands result to be broad and weak (Figure 1(a)). Nevertheless, some differences can be highlighted: in the spectra of the glues, the combination band of asymmetric methylenic $\mathrm{C}-\mathrm{H}$ stretching and bending is located at $4376 \mathrm{~cm}^{-1}$, while in the spectra related to casein and egg white, this absorption band is located at about $4340 \mathrm{~cm}^{-1}$. Furthermore, this absorption band results to be more intense than the symmetric one, always for the case of glues.

The micro-Raman spectra of casein, egg white, and glues (Figure 1(b)) are characterized by the vibrations related to amide I (at about $1665 \mathrm{~cm}^{-1}$ ), $\mathrm{CH}_{2}$ scissoring (at about $1450 \mathrm{~cm}^{-1}$ ), and amide III (at about $1245 \mathrm{~cm}^{-1}$ ). A sharp band due to the aromatic ring breathing is present in all these spectra, which arises from phenylalanine amino acid residues (at about $1002 \mathrm{~cm}^{-1}$ ) [6,18]. This last peak is especially pronounced in egg white and casein, for which phenylalanine is present in higher concentrations than in collagen-based glues [18].

Furthermore, contributions due to tyrosine amino acid are present in all these protein-based binders (826-850, 1210, and $1605-1610 \mathrm{~cm}^{-1}$ ) [18]. Moreover, in the spectra of casein and egg white, peaks ascribable to tryptophan amino acid (at approximately 1550 and $880 \mathrm{~cm}^{-1}$ ) and to phosphoproteins $\left(\sim 954 \mathrm{~cm}^{-1}\right)$ can be found [18].

The FT-NIR spectra related to egg yolk and whole egg, shown in Figure 2(a), are characterized by the methylenic combination bands and their overtones, in the ranges 4250 $4350 \mathrm{~cm}^{-1}$ and $5600-5800 \mathrm{~cm}^{-1}$, more intense with respect to the other protein-based binders, due to the lipid content of 


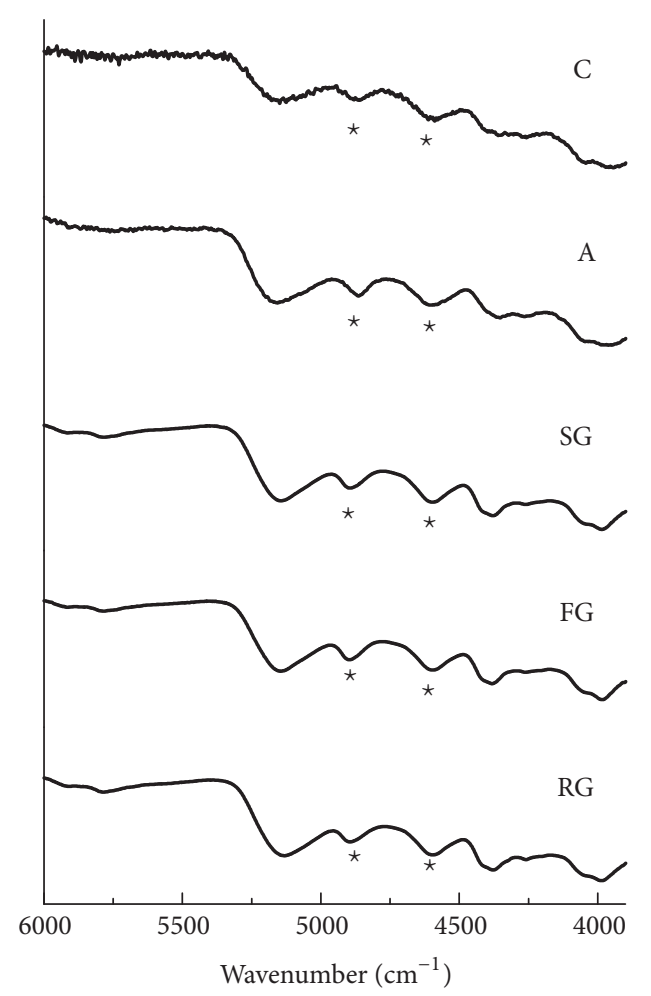

(a)

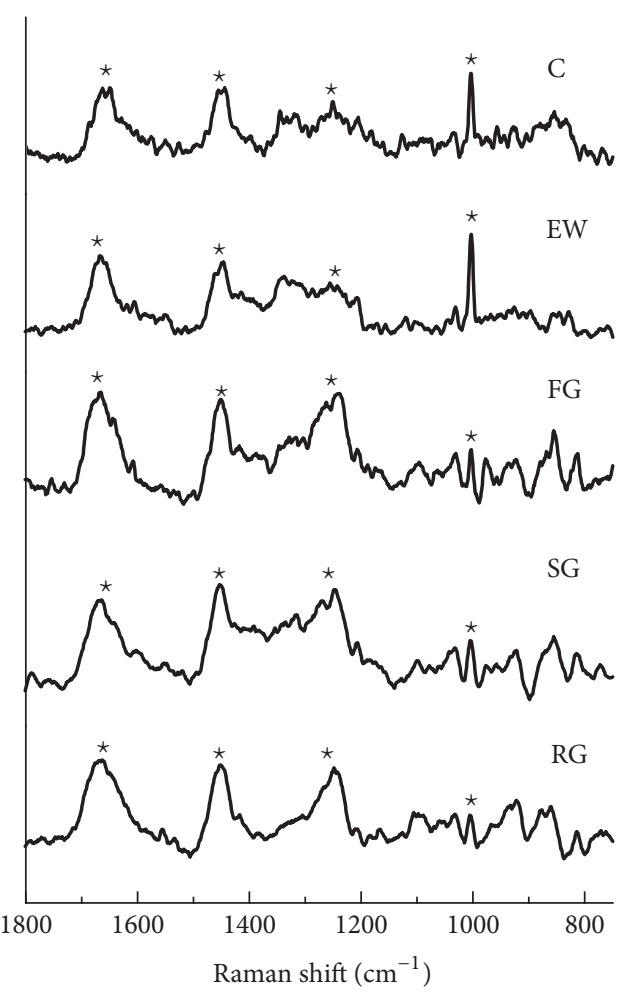

(b)

FIGURE 1: FT-NIR (a) and Raman (b) spectra of casein-C, egg white-EW, fish glue-FG, strong glue-SG, and rabbit glue-RG. Raman spectra are presented after background subtraction. Star symbols point to some specific spectral features discussed in the text. The assignments of the FTNIR and Raman spectra are reported in Tables S1 and S2 in Supplementary Material available online at https://doi.org/10.1155/2017/3475659.

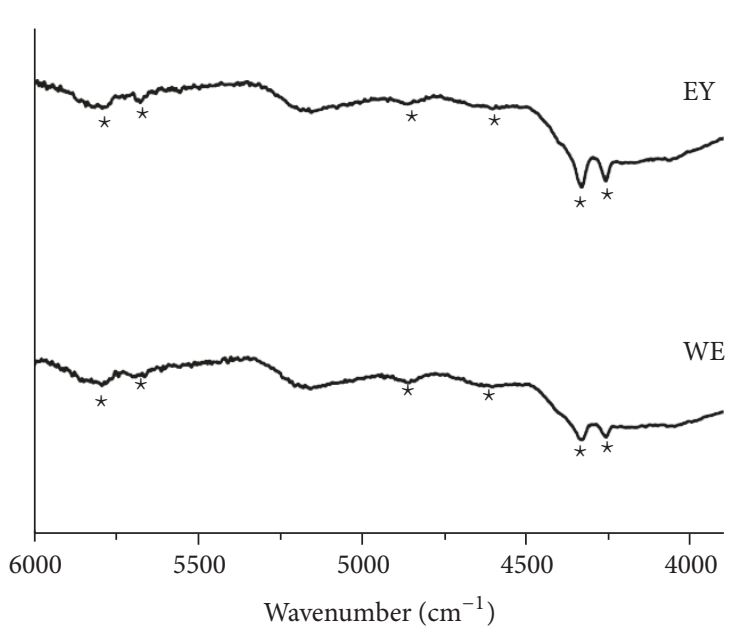

(a)

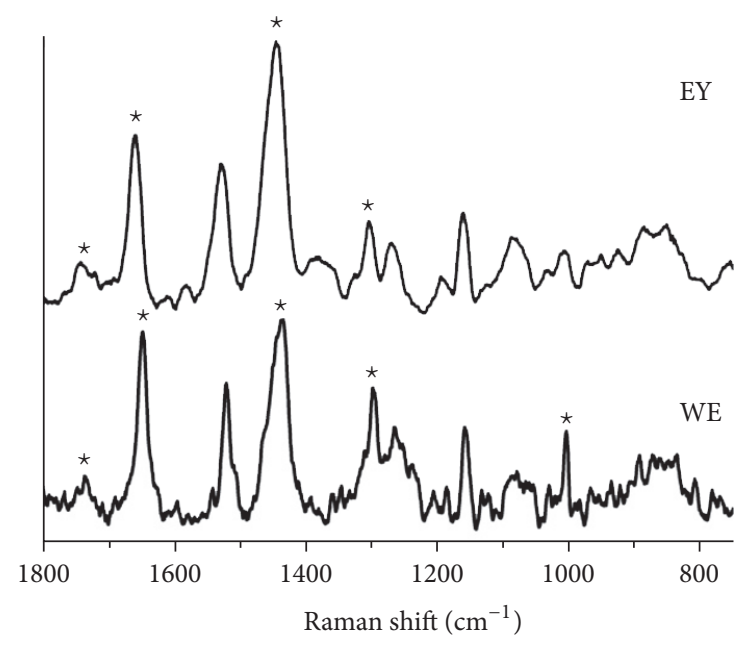

(b)

FIGURE 2: FT-NIR (a) and Raman (b) spectra of egg yolk-EY and whole egg-WE. Raman spectra are presented after background subtraction. Star symbols point to some specific spectral features discussed in the text. The assignments of the FT-NIR and Raman spectra are reported in Tables S3 and S4.

yolk $[6,21]$. The shape and position of the methylenic combination bands from the lipid component in egg yolk/whole egg are different with respect to those from drying oils, thus providing distinctive features for their recognition $[6,21]$.
However, the main proteinaceous bands are all detectable, as can be seen in Figure 2(a).

Concerning the corresponding Raman spectra (Figure 2(b)), due to the presence of fatty acid esters, the characteristic 


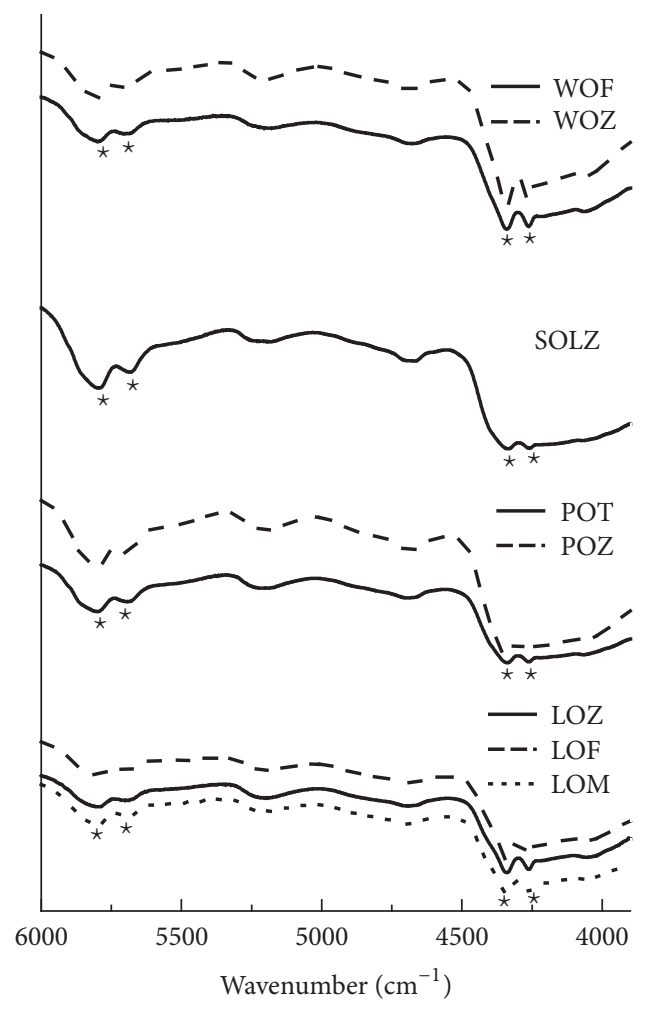

(a)

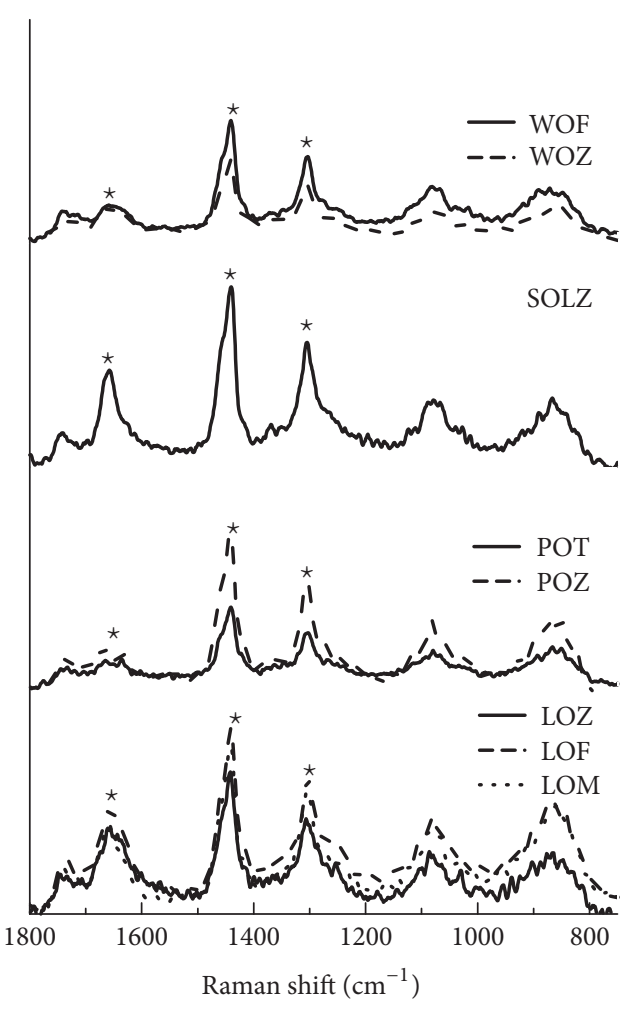

(b)

FIGURE 3: FT-NIR (a) and Raman (b) spectra of drying oils. Raman spectra are presented after background subtraction. Star symbols point to some specific spectral features discussed in the text. Symbols for each drying oil are taken from Table 1. The assignments of the FT-NIR and Raman spectra are reported in Tables S5 and S6.

carbonyl vibration at $1740 \mathrm{~cm}^{-1}$ is present. Furthermore, bands due to the following vibrations are predominant: $1302 \mathrm{~cm}^{-1}$ (in-phase methylene twist) [18], $1442 \mathrm{~cm}^{-1}$ (scissoring mode of methylene) [18], and $1655 \mathrm{~cm}^{-1}$, (cis-double bond stretching) [22]. This last band has a frequency close to the usual value at which the amide I band is observed $\left(1665 \mathrm{~cm}^{-1}\right)[18,22]$. Egg yolk and whole egg have the lowest values for polyunsaturated fatty acids in comparison with drying oils $[2,8]$. Nevertheless, characteristic proteinaceous bands are present, as can be seen in Figure 2(b). The phenylalanine ring breathing $\left(\sim 1002 \mathrm{~cm}^{-1}\right)[18,22]$ results to be more intense in whole egg spectrum, because it presents a major proteinaceous content for the preparation (it was prepared mixing one part of egg white with two parts of egg yolk and one part of vinegar).

FT-NIR spectra of drying oils are shown in Figure 3(a). As regards drying oils, the main near infrared absorption bands are represented by the combination of methylenic C$\mathrm{H}$ stretching and bending (between 4250 and $4350 \mathrm{~cm}^{-1}$ ) and first $\mathrm{CH}_{2}$ stretching overtone bands (in the $5700-5800 \mathrm{~cm}^{-1}$ spectral range). The second overtone of ester carbonyl stretching mode is observed at $5176 \mathrm{~cm}^{-1}$, whereas the combination of ester $\mathrm{C}=\mathrm{O}$ and methylenic $\mathrm{C}-\mathrm{H}$ stretching modes occurs at about $4690 \mathrm{~cm}^{-1}[6,8]$. Raw FT-NIR spectra of drying oils do not show significant spectral differences between each other. In addition, the FT-NIR spectrum related to stand-oil does not show any information that could be ascribed to the effect of the heating process used during its preparation.

On the other hand, the major Raman bands (shown in Figure 3(b)) are represented by symmetric rock in cisdouble bond $\left(\sim 1265 \mathrm{~cm}^{-1}\right)$, in-phase methylene twist mode $\left(\sim 1302 \mathrm{~cm}^{-1}\right)$, scissoring mode of methylene $\left(\sim 1442 \mathrm{~cm}^{-1}\right)$, cis-double bound stretching mode $\left(\sim 1655 \mathrm{~cm}^{-1}\right)$, and ester stretching $\left(\sim 1745 \mathrm{~cm}^{-1}\right)$, as discussed by different authors [2, 8]. Therefore, the drying oils Raman spectra predominantly contain bands arising from vibrations of the hydrocarbon chains. As can be seen in Figure 3(b), the major difference between the different drying oils is located at the band $1655 \mathrm{~cm}^{-1}$ that is more intense for linseed oil and stand-oil. Linseed oil, indeed, contains around $52-55 \%$ of linolenic acid in its chemical composition, whereas poppy-seed oil contains $0.4-0.6 \%$ and walnut oil contains $10-15 \%[2,22]$. Concerning stand-oil, the heating process generates a complex set of reactions leading to different products, including systems with conjugated double bonds [23].

Broad bands characterize the FT-NIR spectra of gums, as shown in Figure 4(a). The characteristic signals of polysaccharides are represented by the combination bands of $\mathrm{O}-\mathrm{H}$ stretching and bending $\left(\sim 5140 \mathrm{~cm}^{-1}\right)$, of $\mathrm{O}-\mathrm{H}$ bending and C-O stretching $\left(\sim 4800 \mathrm{~cm}^{-1}\right)$ and of $\mathrm{C}-\mathrm{H}$ and $\mathrm{C}-\mathrm{C} / \mathrm{C}-\mathrm{O}-\mathrm{C}$ stretching modes $\left(\sim 4010 \mathrm{~cm}^{-1}\right)$ [21]. In addition, a very broad 


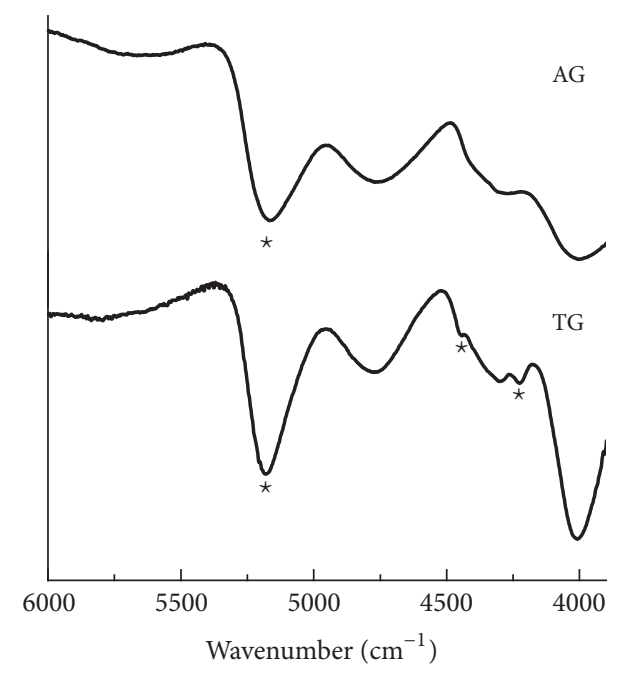

(a)

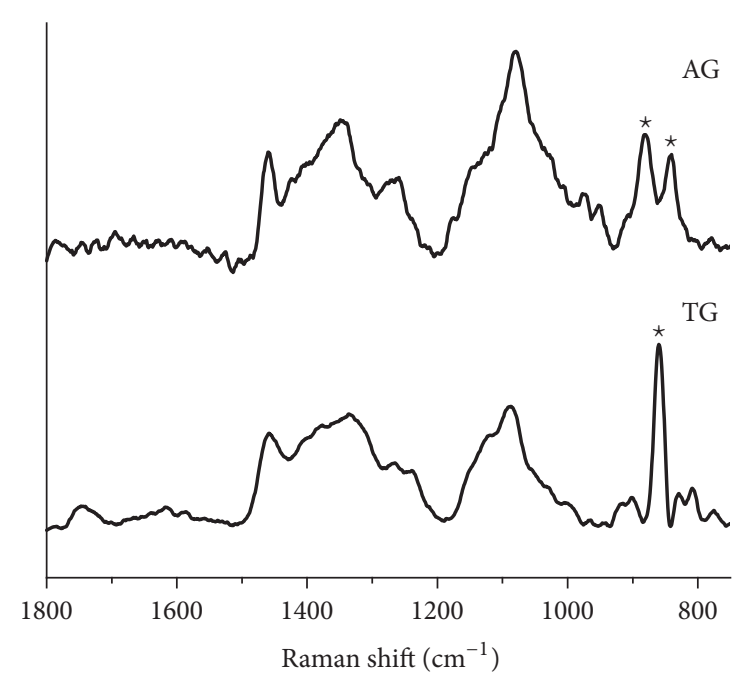

(b)

FIGURE 4: FT-NIR (a) and Raman (b) spectra of tragacanth gum-TG and Arabic gum-AG. Raman spectra are presented after background subtraction. Star symbols point to some specific spectral features discussed in the text. The assignments of the FT-NIR and Raman spectra are reported in Tables S7 and S8.

band ascribed to combination band of $\mathrm{C}-\mathrm{H}$ stretching and bending, at about $4270 \mathrm{~cm}^{-1}$, can be observed [21].

As regards the near infrared region, the combination band of O-H stretching and bending represents the main difference between two gums. In the spectrum of tragacanth gum, this band is shifted of around $20 \mathrm{~cm}^{-1}$ towards longer wavenumbers in comparison with Arabic gum.

The Raman spectra, shown in Figure 4(b), do not present intense peaks in the $1800-1500 \mathrm{~cm}^{-1}$ spectral range. This feature already differentiates the gums from the other binding media previously described. These materials, indeed, do not contain aromatic or aliphatic $\mathrm{C}=\mathrm{C}$ double bonds. In addition, no amide I and amide III bands are present, in contrast to proteinaceous binders $[22,24]$. The rather broad peaks in the $1500-1200 \mathrm{~cm}^{-1}$ region are ascribed to C$\mathrm{H}$ deformations, whereas the peaks at lower wavenumbers are associated with $\mathrm{C}-\mathrm{C}$ and $\mathrm{C}-\mathrm{O}$ symmetrical stretching modes $\left(1200-950 \mathrm{~cm}^{-1}\right)$ and C-O-C sugar ring vibrations $\left(950-800 \mathrm{~cm}^{-1}\right)[22,24]$. The Raman spectrum of Arabic gum shows a doublet at around $850 \mathrm{~cm}^{-1}\left(882 \mathrm{~cm}^{-1}\right.$ and $\left.841 \mathrm{~cm}^{-1}\right)$, whereas tragacanth gum has a singlet $\left(857 \mathrm{~cm}^{-1}\right)$. This feature allows distinguishing between the two gums.

\subsection{PCA Applied on Combined First Derivative FT-NIR and} Micro-Raman Spectra. In this section, the potentialities of PCA to improve the description of each class of binders are evaluated and discussed.

The application of a multivariate analytical tool, such as PCA, to the combined first derivative FT-NIR and Raman spectra allows the concomitant extraction of important information. The obtained statistical model explains a significant percentage (83\%) of the cumulative variability by means of the first four PCs. In detail, PC1 explains $49 \%$ of the total variance contained in the data, whereas $\mathrm{PC} 2, \mathrm{PC} 3$, and PC4 explain 19,11 , and $4 \%$ of the total variance, respectively.
Focusing on the two-dimensional score plot in the space defined by PC1 and PC2 (Figure 5) four different data groups can be individuated: (1) gums; (2) drying oils; (3) egg yolk and whole egg; (4) glues, casein, and egg white.

In Figures 6(a), 6(b), and 6(c), the loading plots of $\mathrm{PC} 1$ and PC2 are reported, highlighting the contributions that can be ascribed to polysaccharide-, lipid-, and proteinbased binding media, respectively. In all loading plots, the "plus" symbol is associated with a first derivative contribution concordant with the starting combined spectra, while a "minus" sign is used when its behavior is reversed. Gums dataset displays a negative score value for $\mathrm{PCl}$, whereas it has a positive score value for PC2 (Figure 5). As can be seen in Figure 6(a), the greatest contributions to PC1 related to polysaccharide-based binding media are represented by the combination band of $\mathrm{O}-\mathrm{H}$ stretching and bending $(\sim$ $5140 \mathrm{~cm}^{-1}$ ) [21], the combination band of $\mathrm{C}-\mathrm{H}$ and $\mathrm{C}-\mathrm{C} / \mathrm{C}-$ O-C stretching modes $\left(\sim 4010 \mathrm{~cm}^{-1}\right)$ [21], and the C-O-C sugar ring vibrations $\left(950-800 \mathrm{~cm}^{-1}\right)[22,24]$. As regards PC2, the main highlighted polysaccharide vibrations are the combination band of $\mathrm{O}-\mathrm{H}$ stretching and bending and the C-O-C sugar ring vibrations (Figure 6(a)). So, the first two PCs allow describing polysaccharide materials, immediately focusing on their principal spectral properties in NIR and Raman regions but do not allow any further differentiation.

Focusing, instead, on drying oils, these binders datasets contribute with a positive score value to $\mathrm{PCl}$, whereas they result to be very close to a zero value for PC2 (Figure 5). PC1, shown in Figure 6(b), is characterized by bands associated with drying oils, such as the $\mathrm{CH}_{2}$ stretching first overtone band (in the $5700-5800 \mathrm{~cm}^{-1}$ spectral range) $[6,8]$, combination band of methylenic $\mathrm{C}-\mathrm{H}$ stretching and bending (between 4250 and $4350 \mathrm{~cm}^{-1}$ ) $[6,8]$, scissoring mode of methylene $\left(1442 \mathrm{~cm}^{-1}\right)[2,8]$, and in-phase methylene twist mode $\left(\sim 1302 \mathrm{~cm}^{-1}\right)[2,8]$. In PC2, their main contributions 


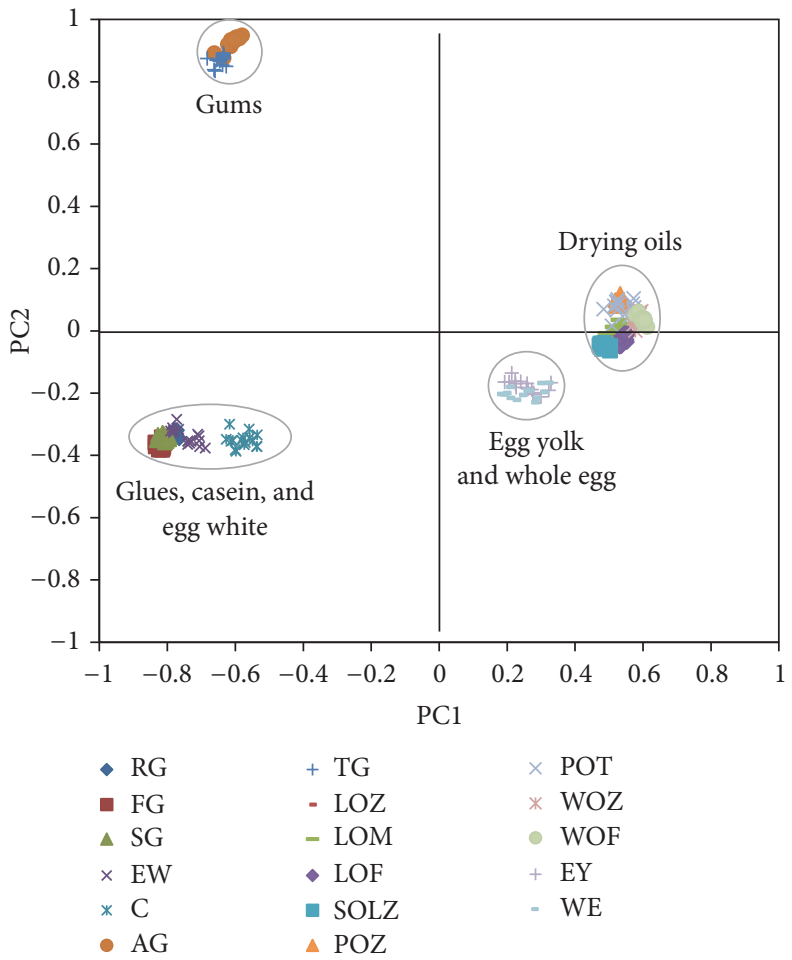

FIGURE 5: PCA applied to combined FT-NIR and Raman first derivative spectra, taking into account all neat binding media: score plot of PC1-PC2.

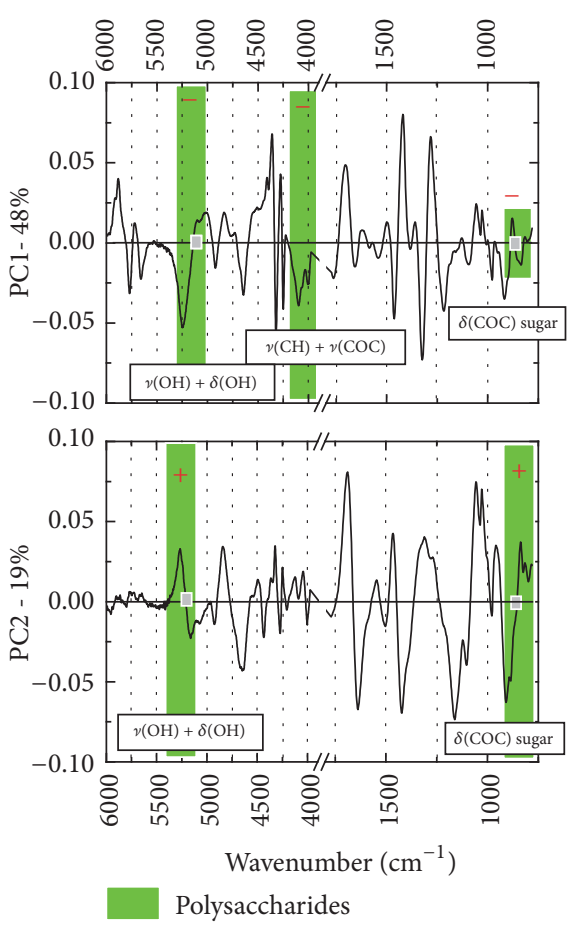

(a)

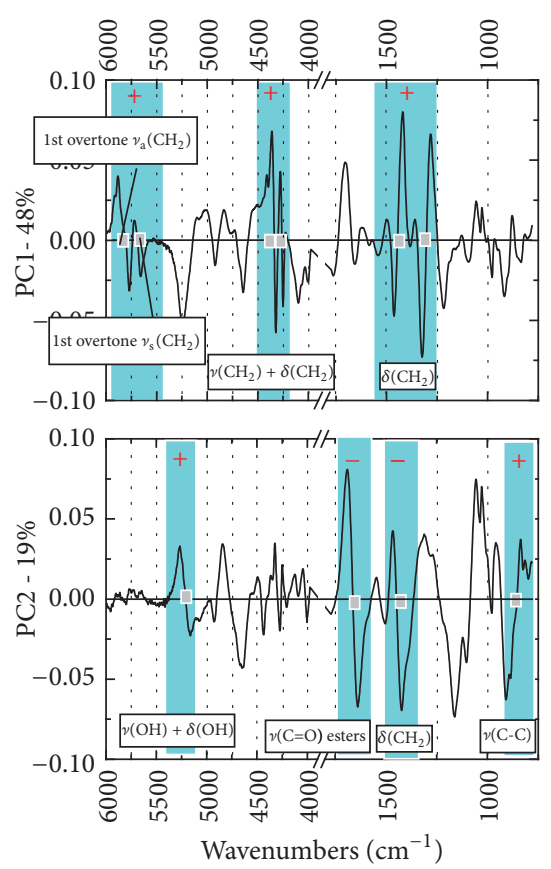

Lipidic binders

(b)

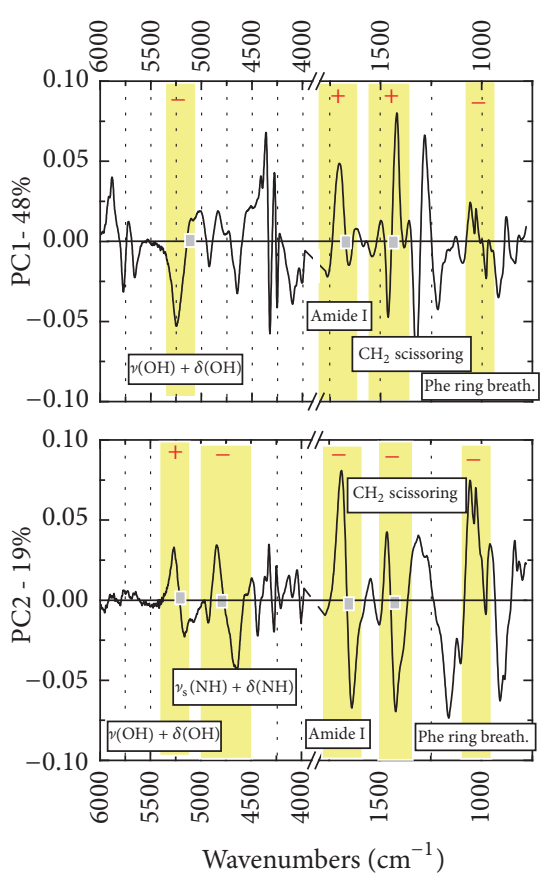

Proteinaceous binders

(c)

FIGURE 6: PCA applied to combined FT-NIR and Raman first derivative spectra, taking into account all neat binding media: score plot of PC1-PC2 (a); loading plots of PC1 and PC2, highlighting in the different panels the bands associated with different materials: polysaccharides (a), lipids (b), and proteinaceous materials (c). 


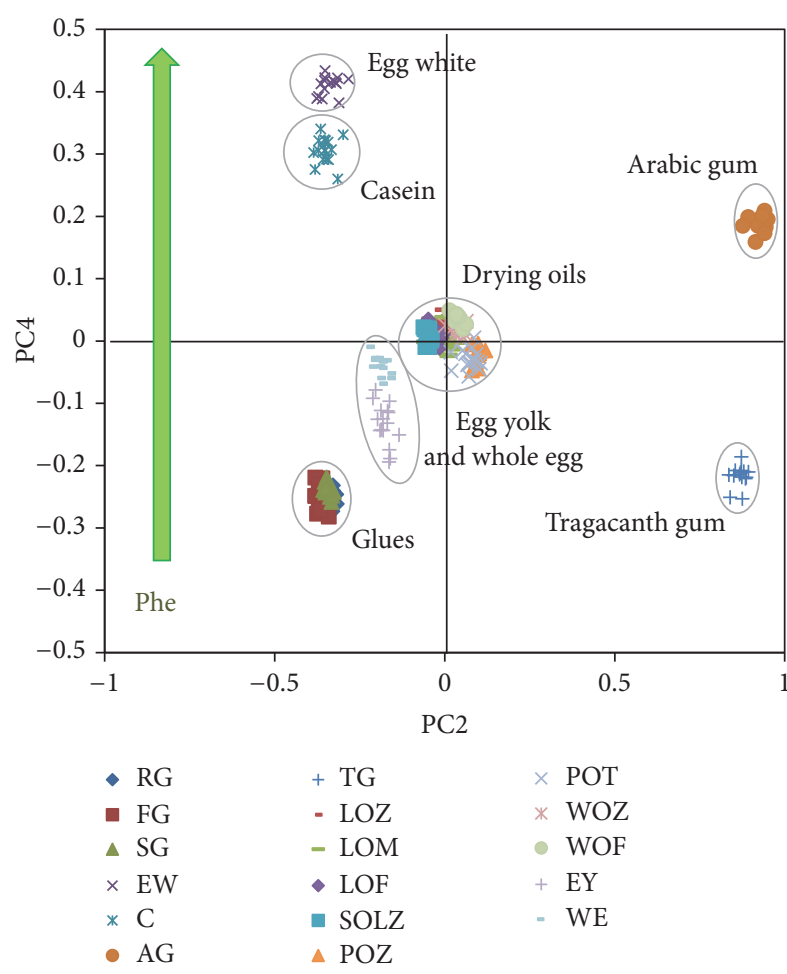

(a)

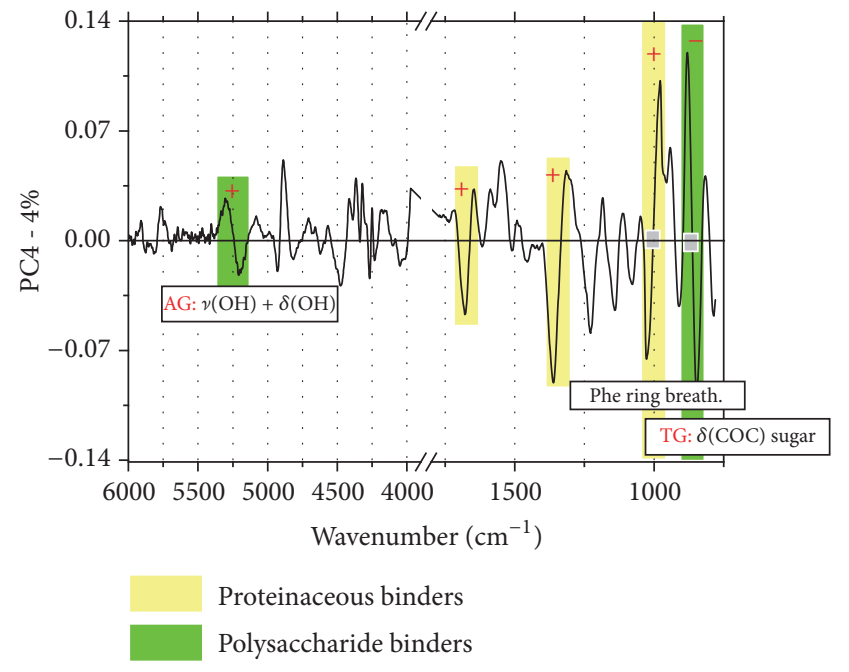

(b)

FIGURE 7: PCA applied to combined FT-NIR and Raman spectra, taking into account all neat binding media: score plot of PC2-PC4 (a) and loading plot of PC4 (b), highlighting proteinaceous and polysaccharide contributions.

are related to combination band of methylenic $\mathrm{C}-\mathrm{H}$ stretching and bending, in NIR region, and scissoring mode of methylene, in Raman region. These contributions cause a very low score value for PC2, because they are opposed: the former is positive, while the latter is negative (Figure 6(b)). Also, for drying oils this statistical model immediately allows to highlight their characteristic spectral bands. Nevertheless, no differentiation between various drying oils is obtained.

Concerning glues, $\mathrm{C}$, and EW display a negative score value for PC1 and a negative one for PC2 (Figure 5). As regards proteinaceous binders, in the loading plot of PC1 (Figure 6(c)), specific contributions due to amide I $\left(\sim 1665 \mathrm{~cm}^{-1}\right)$, $\mathrm{CH}_{2}$ scissoring $\left(\sim 1450 \mathrm{~cm}^{-1}\right)$, and phenylalanine ring breathing $\left(1002 \mathrm{~cm}^{-1}\right)$ vibrations can be observed $[18,22]$. Nevertheless, PC2 better describes protein-based binding media. Indeed, in the corresponding loading plot (Figure 6(c)) intense contributions related to the combination band of the stretching and bending modes of $\mathrm{NH}$ at about $4880 \mathrm{~cm}^{-1}$, amide I mode, $\mathrm{CH}_{2}$ scissoring vibration, and phenylalanine ring breathing mode can be highlighted $[6,21]$. Instead, EY and WE are located at an intermediate position between drying oils and the other protein-based binding media (Figure 5). These binders are characterized by a lipid content that is completely absent in C, EW, and glues.

As previously discussed, the purpose of all multivariate data analysis is to decompose the data in order to detect and model the "hidden phenomena." Taking into mind this principle/precept, the PC4 results to be notably interesting. As we can observe in the space defined by the score plot of PC2 and PC4 (Figure 7(a)) a clear separation between AG and TG can be observed as well as between EW, C, and glues. Indeed, the loading plot of PC4, reported in Figure $7(\mathrm{~b})$, is characterized by two main contributions: phenylalanine ring breathing and $\mathrm{C}-\mathrm{O}-\mathrm{C}$ ring vibration. Concerning phenylalanine ring breathing vibration, PC4 defines a trend that describes the different content of this amino acid in proteinaceous binding media. The peak at $1002 \mathrm{~cm}^{-1}$, as previously described, is especially pronounced in EW and C Raman spectra, for which phenylalanine is present in higher concentrations than in collagen-based glues (a total of approximately $12 \%$ of tyrosine plus phenylalanine is detected in egg and casein, compared to approximately $4 \%$ total in animal glue) [18].

On the other hand, C-O-C ring vibration, also described by PC4, shows the specific frequency related to TG: the characteristic singlet at $857 \mathrm{~cm}^{-1}[22,24]$. Additionally, another minor contribution, ascribable to gums, is the combination band of $\mathrm{OH}$ stretching and bending. In this case, the corresponding frequency is $5180 \mathrm{~cm}^{-1}$, which is close to that observed for AG near infrared spectrum (Figure 4(a)).

In Table 2 a summary of the main spectral properties highlighted by this statistical model for the different classes of binding media is reported.

\section{Conclusions}

In this work, we have analyzed model films of protein-, polysaccharide-, and lipid-based binding media after a natural 
TABLE 2: Main near infrared and Raman bands, characteristic for different classes of binding media, highlighted by PCA applied on combined spectra referred to all neat binders.

\begin{tabular}{lcc}
\hline Class of binders & FT-NIR & Micro-Raman \\
\hline Polysaccharide-based & $\begin{array}{c}\nu(\mathrm{OH})+\delta(\mathrm{OH}) \\
\nu(\mathrm{CH})+\nu(\mathrm{COC})\end{array}$ & $\begin{array}{c}\delta(\mathrm{COC}) \text { sugar ring } \\
\text { vibrations }\end{array}$ \\
\hline \multirow{2}{*}{ Lipid-based } & $\begin{array}{l}1 \text { st overtone } \mathrm{CH}_{2} \\
\nu\left(\mathrm{CH}_{2}\right)+\delta\left(\mathrm{CH}_{2}\right)\end{array}$ & $\delta\left(\mathrm{CH}_{2}\right)$ \\
& {$[\nu(\mathrm{OH})+\nu(\mathrm{OH})]$} & \\
\hline \multirow{2}{*}{ Protein-based } & $\nu(\mathrm{NH})+\delta(\mathrm{NH})$ & $\begin{array}{c}\text { Amide I } \\
\delta\left(\mathrm{CH}_{2}\right)\end{array}$ \\
& $\nu(\mathrm{OH})+\nu(\mathrm{OH})$ & Phe ring breathing \\
\hline
\end{tabular}

ageing of three months, highlighting the applicability of FTNIR and Raman spectroscopies for their characterization. Assignment of main near infrared and Raman bands revealed significant differences between spectra related to various classes of binders. These differences are emphasized by PCA applied on the corresponding combined first derivative spectra. The application of PCA on combined first derivative spectra has allowed immediately visualizing the main spectral properties, in both regions investigated, of these materials. The proposed method represents a novel noninvasive and nondestructive approach for their identification and differentiation.

Additionally, the results described highlighted the capability of this methodology to extract information related to hidden variance. Therefore, we differentiated egg white and casein between each other and from glues, based on different content in the amino acid phenylalanine. It has been also possible to distinguish between Arabic gum and tragacanth gum. Nevertheless, we did not observe any differentiation within drying oils or glues groups. This result indicates and suggests the need for further PCA modelling of each such group in order to fine-tune the analysis and to understand the characteristic features of the different classes of binders.

This work represents a contribution to the development of nondestructive, optical methods that could provide knowledge of the binder media used by artists. Their identification is often quite difficult due to their complex molecular nature. The development of these methods, which could act without detriment of the sample, helps not only in complementing historical research, but also in the process of conservation and restoration of artworks.

\section{Conflicts of Interest}

The authors declare that there are no conflicts of interest regarding the publication of this article.

\section{Acknowledgments}

Authors wish to thank Dr. M. Picollo, IFAC-CNR, Italy, for making available the ALPHA-FTIR spectrometer for this research. This work was supported by Italian MIUR (PRIN, Grant 2010329WPF_007) and by University of Florence (ex60\%). Support from Ente Cassa di Risparmio di Firenze (Grant no. 2014.0405A2202.8044) is also kindly acknowledged.

\section{References}

[1] B. H. Stuart, Analytical Techniques in Materials Conservation, John Wiley \& Sons, New York, NY, USA, 2007.

[2] E. Manzano, J. García-Atero, A. Dominguez-Vidal, M. J. AyoraCañada, L. F. Capitán-Vallvey, and N. Navas, "Discrimination of aged mixtures of lipidic paint binders by Raman spectroscopy and chemometrics," Journal of Raman Spectroscopy, vol. 43, no. 6, pp. 781-786, 2012.

[3] M. P. Colombini, A. Andreotti, I. Bonaduce, F. Modugno, and E. Ribechini, "Analytical strategies for characterizing organic paint media using gas chromatography/mass spectrometry," Accounts of Chemical Research, vol. 43, no. 6, pp. 715-727, 2010.

[4] N. Navas, J. Romero-Pastor, E. Manzano, and C. Cardell, "Raman spectroscopic discrimination of pigments and tempera paint modelsamples by principal component analysis on firstderivative spectra," Journal of Raman Spectroscopy, vol. 41, no. 11, pp. 1486-1493, 2010.

[5] N. Navas, J. Romero-Pastor, E. Manzano, and C. Cardell, "Benefits of applying combined diffuse reflectance FTIR spectroscopy and principal component analysis for the study of blue tempera historical painting," Analytica Chimica Acta, vol. 630, no. 2, pp. 141-149, 2008.

[6] M. Vagnini, C. Miliani, L. Cartechini, P. Rocchi, B. G. Brunetti, and A. Sgamellotti, "FT-NIR spectroscopy for non-invasive identification of natural polymers and resins in easel paintings," Analytical and Bioanalytical Chemistry, vol. 395, no. 7, pp. 21072118, 2009.

[7] A. Pallipurath, J. Skelton, P. Ricciardi, S. Bucklow, and S. Elliott, "Multivariate analysis of combined Raman and fibre-optic reflectance spectra for the identification of binder materials in simulated medieval paints," Journal of Raman Spectroscopy, vol. 44, no. 6, pp. 866-874, 2013.

[8] S. Carlesi, M. Ricci, C. Cucci et al., "Multivariate analysis of combined fourier transform near-infrared spectrometry (FTNIR) and Raman datasets for improved discrimination of drying oils," Applied Spectroscopy, vol. 69, no. 7, pp. 865-876, 2015.

[9] S. Carlesi, M. Ricci, C. Cucci, C. Lofrumento, M. Picollo, and M. Becucci, "Multivariate analysis of combined reflectance FT-NIR and micro-Raman spectra on oil-paint models," Microchemical Journal, vol. 124, pp. 703-711, 2016.

[10] J. Bueno and I. K. Lednev, "Advanced statistical analysis and discrimination of gunshot residue implementing combined Raman and FT-IR data," Analytical Methods, vol. 5, no. 22, pp. 6292-6296, 2013.

[11] A. Zoppi, C. Lofrumento, M. Ricci, E. Cantisani, T. Fratini, and E. M. Castellucci, "A novel piece of Minoan art in Italy: the first spectroscopic study of the wall paintings from Phaistos," Journal of Raman Spectroscopy, vol. 43, no. 11, pp. 1663-1670, 2012.

[12] J. Romero-Pastor, N. Navas, S. Kuckova, A. Rodríguez-Navarro, and C. Cardell, "Collagen-based proteinaceous binder-pigment interaction study under UV ageing conditions by MALDITOF-MS and principal component analysis," Journal of Mass Spectrometry, vol. 47, no. 3, pp. 322-330, 2012.

[13] C. Pasquini, "Near infrared spectroscopy: fundamentals, practical aspects and analytical applications," Journal of the Brazilian Chemical Society, vol. 14, no. 2, pp. 198-219, 2003. 
[14] M. Otto, Chemometrics: Statistics and Computer Application in Analytical Chemistry, John Wiley \& Sons, 2007.

[15] K. H. Esbensen, "Multivariate Data Analysis-In Practice," CAMO Software AS., 2009.

[16] C. Cennini and F. Frezzato, "Il Libro Dell'arte," N. Pozza, 2003.

[17] L. Masschelein-Kleiner, Ancient Binding Media, Varnishes and Adhesives, ICCROM, Rome, Italy, 2nd edition, 1995.

[18] A. Nevin, I. Osticioli, D. Anglos, A. Burnstock, S. Cather, and E. Castellucci, "Raman spectra of proteinaceous materials used in paintings: a multivariate analytical approach for classification and identification," Analytical Chemistry, vol. 79, no. 16, pp. 6143-6151, 2007.

[19] I. Bonaduce, L. Carlyle, M. P. Colombini et al., "New insights into the ageing of linseed oil paint binder: a qualitative and quantitative analytical study," PLoS ONE, vol. 7, no. 11, Article ID e49333, 2012.

[20] A. Savitzky and M. J. E. Golay, "Smoothing and differentiation of data by simplified least squares procedures," Analytical Chemistry, vol. 36, no. 8, pp. 1627-1639, 1964.

[21] D. A. Burns and E. W. Ciurczak, Handbook of Near-infrared Analysis, CRC Press, Boca Raton, Fla, USA, 2008.

[22] P. Vandenabeele, B. Wehling, L. Moens, H. Edwards, M. De Reu, and G. Van Hooydonk, "Analysis with micro-Raman spectroscopy of natural organic binding media and varnishes used in art," Analytica Chimica Acta, vol. 407, no. 1-2, pp. 261$274,2000$.

[23] O. Zovi, L. Lecamp, C. Loutelier-Bourhis, C. M. Lange, and C. Bunel, "Stand reaction of linseed oil," European Journal of Lipid Science and Technology, vol. 113, no. 5, pp. 616-626, 2011.

[24] L. Brambilla, C. Riedo, C. Baraldi et al., "Characterization of fresh and aged natural ingredients used in historical ointments by molecular spectroscopic techniques: IR, Raman and fluorescence," Analytical and Bioanalytical Chemistry, vol. 401, no. 6, pp. 1827-1837, 2011. 

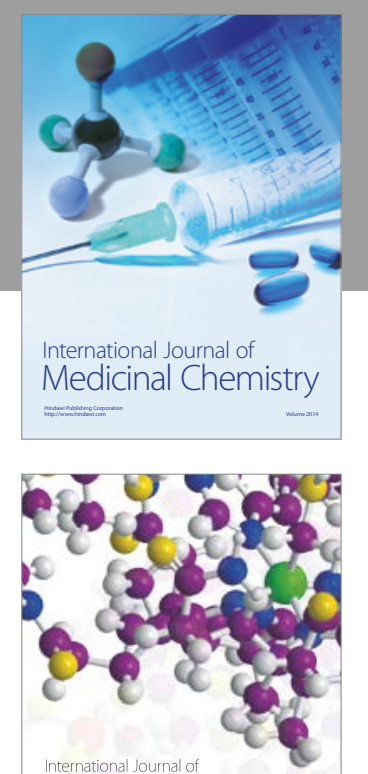

Carbohydrate Chemistry

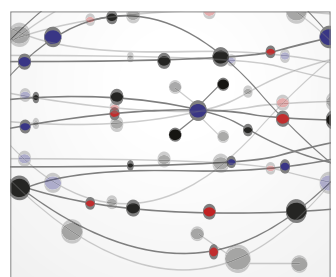

The Scientific World Journal
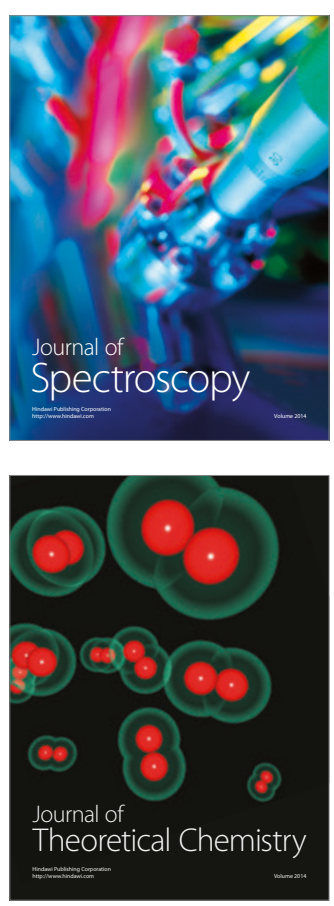
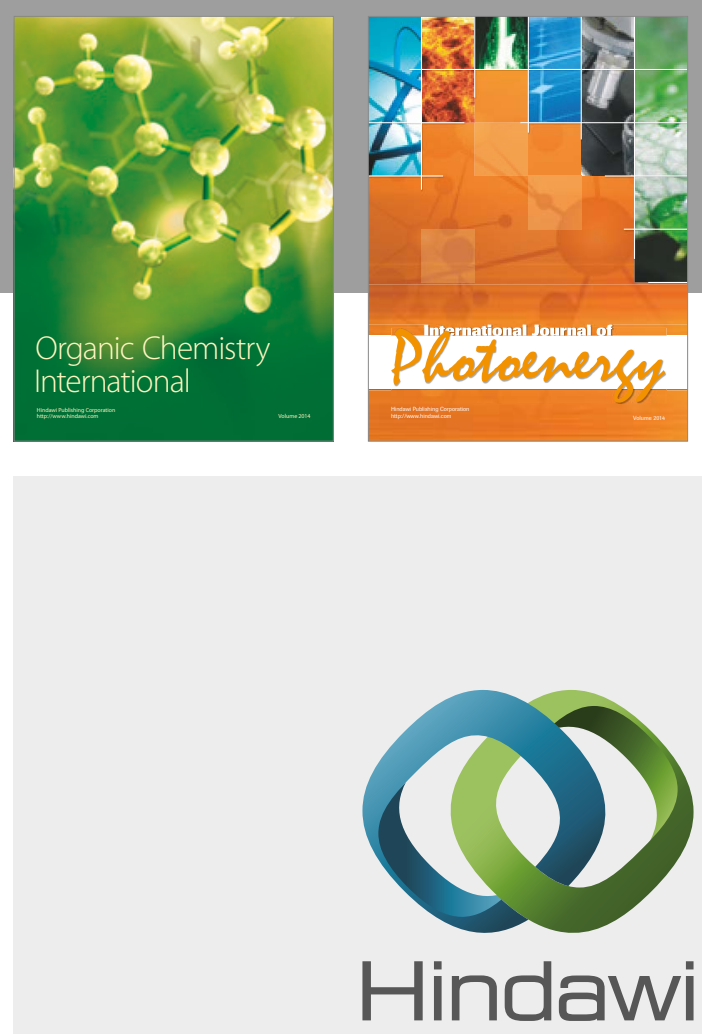

Submit your manuscripts at

https://www.hindawi.com

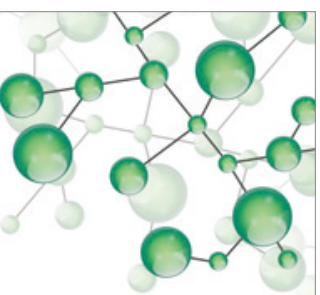

International Journal of

Inorganic Chemistry

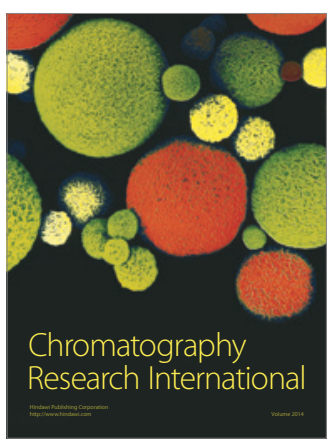

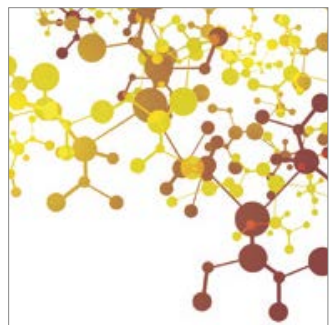

Applied Chemistry
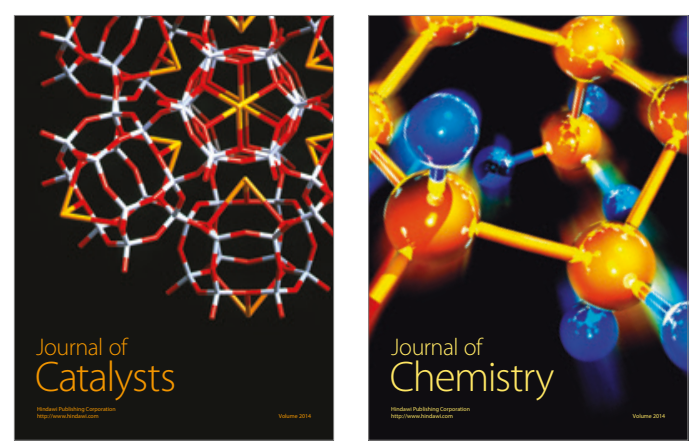
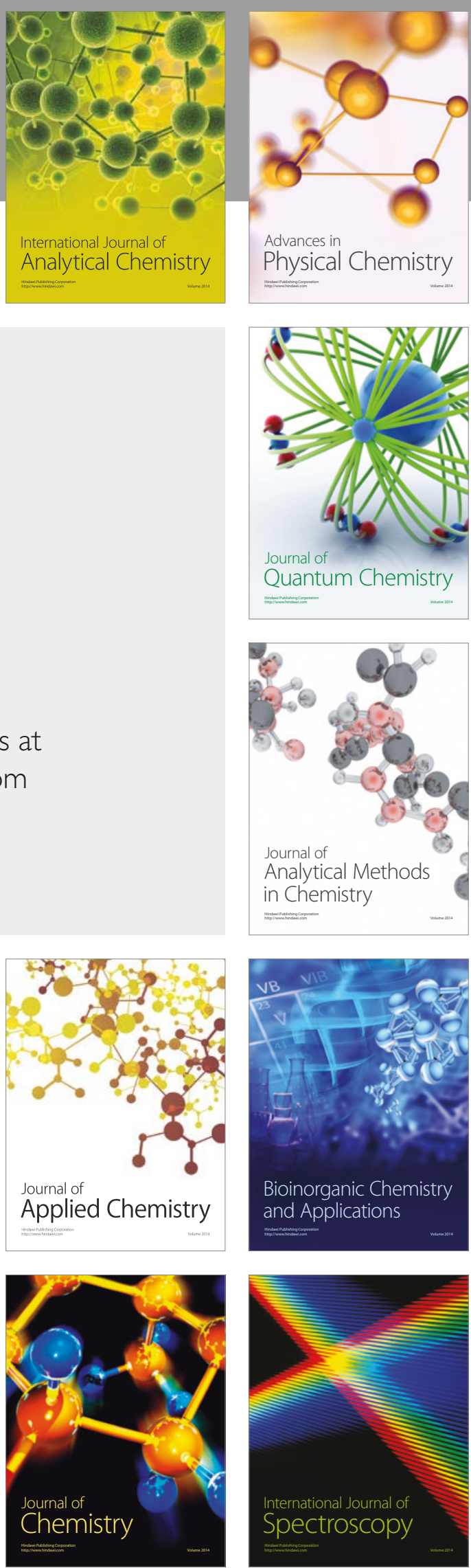